\title{
Article \\ On the Estimation of the Moving Mass of a TMD Installed on a Lively Structure
}

\author{
Alvaro Magdaleno*(D), Cesar Pelaez $(\mathbb{D}$, Alvaro Iglesias-Pordomingo and Antolin Lorenzana \\ ITAP, School of Industrial Engineering, University of Valladolid, Paseo del Cauce 59, 47011 Valladolid, Spain; \\ cesar.pelaez@uva.es (C.P.); alvaro.iglesias@alumnos.uva.es (A.I.-P.); ali@eii.uva.es (A.L.) \\ * Correspondence: alvaro.magdaleno@uva.es
}

check for updates

Citation: Magdaleno, A.; Pelaez, C.; Iglesias-Pordomingo, A.; Lorenzana, A. On the Estimation of the Moving Mass of a TMD Installed on a Lively Structure. Appl. Sci. 2021, 11, 4712. https://doi.org/10.3390/app11104712

Academic Editor: Antonella

D’Alessandro

Received: 6 April 2021

Accepted: 19 May 2021

Published: 20 May 2021

Publisher's Note: MDPI stays neutral with regard to jurisdictional claims in published maps and institutional affiliations.

Copyright: (c) 2021 by the authors. Licensee MDPI, Basel, Switzerland. This article is an open access article distributed under the terms and conditions of the Creative Commons Attribution (CC BY) license (https:// creativecommons.org/licenses/by/ $4.0 /)$.

\begin{abstract}
Tuned Mass Dampers are devices which can be assimilated to single-degree-of-freedom systems with a certain amount of moving mass, a natural frequency and a damping ratio intended to be installed on lively structures to reduce the contribution of a certain mode to their response. Once placed on the structure, the movement of the mass damper couples to the structural response and determines its properties as an isolated system becomes challenging. The authors have previously presented a methodology to estimate the natural frequency and damping ratio of an SDOF system installed on a structure and not necessarily tuned to a certain mode. It was based on a transmissibility function and, thus, the moving mass could not be estimated. With this work, the authors go one step further and present a novel procedure to estimate the moving mass value by means of the same transmissibility function and two well selected frequency response functions. The methodology is applied to estimate the properties of a real single-degree-of-freedom system placed on a lively timber platform. The results are compared with the mass modification technique to show that the proposed methodology provides better estimations in a more efficient way.
\end{abstract}

Keywords: structural dynamics; lively structure; tuned mass damper; system identification

\section{Introduction}

In the field of structural dynamics, tuned mass dampers (TMDs) are passive devices installed on lively structures to reduce their response level [1,2]. They are extensively used not only to mitigate vertical vibrations in, for example, light floors [3] or footbridge decks [4,5], but also horizontal vibration of tall buildings, such as wind turbines [6], chimneys [7], bridge piles or skyscrappers [4,8]. One of the most widespread ways of modeling TMDs is as single-degree-of-freedom (SDOF) systems composed of a moving mass $m_{t}$, an elastic member $k_{t}$ which attaches it to the structure and a damping element $c_{t}$ which accounts for the energy dissipation, conferring it a natural frequency $\omega_{t}=\sqrt{k_{t} / m_{t}}$ and a damping ratio $\zeta_{t}=c_{t} /\left(2 \omega_{t} m_{t}\right)$ as an isolated system [9]. After its installation, and if the device is properly tuned, the movement of both systems is coupled so the kinetic energy flows from one device to another and, during this energy exchange, a part of it is dissipated in the damping element. For the coupling to be effective, the mass damper must be properly tuned, i.e., its natural frequency $\omega_{t}$ must be close to and below one of the natural frequencies of the structure $[9,10]$. In this sense, the TMD only reduces the response of the structure in a relatively narrow frequency band in the vicinity of the affected mode. If the contribution of several modes needs to be mitigated, then several TMDs should be installed or other more sophisticated approaches (active mass dampers, etc.) should be considered. The overall effectiveness of a TMD, as well as the width of the frequency band it affects, can be controlled by means of the amount of moving mass $m_{t}$ : the greater the moving mass, the more effective it is and the wider the affected frequency band. However, since larger moving masses usually involve higher costs, the minimum one fulfilling certain criteria is commonly installed. 
As mentioned above, after the installation of a TMD, its movement is coupled to the structural response, making it difficult to estimate its properties as an isolated device $\left(m_{t}, \omega_{t}, \zeta_{t}\right)$. Even if these properties have been precisely estimated before its installation, it is not possible knowledge of them later, for several reasons. In the short term, the properties of a TMD may be affected by the installation process, which usually involves disassembling and reassembling some of its parts. In the long term, the properties may be modified due to the unavoidable wearing that affects the different elements over time. One common way to cope with these issues is to install multiple TMDs with close tuning frequencies $[11,12]$. Together, these TMDs cover a wider frequency range in comparison to a single device, so the potential structural modifications or detuning issues are mitigated to some extent. However, installing more than one TMD may not be possible in some structures (for its cost, location issues, etc.), and a well-tuned mass damper is more suitable. The most common, but indirect, strategy to evaluate the goodness of the tuning consists in estimating the properties $[13,14]$ or the frequency response of the ensemble (structure with TMD) [15]. By assessing the modal properties (natural frequency and damping ratio) or the amplitude of the frequency response of the structure in the vicinity of the mitigated mode, the tuning of the TMD can be evaluated and, if required, an iterative re-tuning process may be carried out. However, although useful, none of these procedures provide the sought TMD properties by themselves.

The authors of this work already presented a direct transmissibility-based methodology to accurately estimate both the natural frequency $\omega_{t}$ and the damping ratio $\zeta_{t}$ of a TMD installed on a slender structure [16], which works not only for properly tuned mass dampers, but also for any kind of SDOF system installed on a structure, not necessarily tuned to mitigate the contribution of a specific mode. The procedure consists in simultaneously measuring both the acceleration of the TMD moving mass, $\ddot{x}_{t}$, and the acceleration of the point of the structure to which it is attached, $\ddot{x}_{s}$, and estimating the frequency response function between them, called transmissibility function. Two approaches were presented: one in which the frequency response function was directly estimated between the two measured signals and another in which the frequency response function was estimated between the structure acceleration and the relative acceleration $\left(\ddot{x}_{t}-\ddot{x}_{s}\right)$. By fitting the data of each frequency response function to the corresponding transmissibility analytical expression, the natural frequency $\omega_{t}$ and the damping ratio $\zeta_{t}$ were estimated.

In this work, a novel methodology is presented to simultaneously estimate the natural frequency, $\omega_{t}$, the damping ratio, $\zeta_{t}$, and the moving mass, $m_{t}$, of a TMD installed on a lively structure. The major difference between this and the previous work [16] is that the presented procedure enables an estimate of the moving mass of the TMD, $m_{t}$, to be made, something that could not be done before due to the adopted approach being completely based on transmissibility functions. Now, a two-step methodology is presented. The first step is still based on the estimation of a transmissibility function, but the procedure is completed with a second step in which a couple of frequency response functions are calculated by applying a force on the moving mass. These functions, together with the transmissibility, lead to a curve that can be fitted to the classical frequency response function of an SDOF system, which is explicitly expressed in terms of the three parameters of the system, including its moving mass, $m_{t}$. As will be shown, the methodology is entirely based on simple expressions, deduced from the SDOF system theory, so it is within the grasp of every mechanical engineer. In addition, it requires a very reduced set of measurements to be performed, leading to a procedure that is very easy and fast to apply. This constitutes a major advantage with respect to others, such as the mass modification technique. In order to compare the quality of the obtained results against the required workload, that technique is also covered in this article. It is a widespread method also conceived to estimate the moving mass of an SDOF system based on the definition of the undamped natural frequency of an SDOF system.

As mentioned above, the presented methodology is aimed at estimating, in an easy and rapid way, the whole set of modal parameters (moving mass, natural frequency and 
damping ratio) of an installed TMD, which is helpful in a variety of scenarios. It is useful, for example, for the fine tuning of the mass damper in order to overcome modifications it may suffer while assembling or transporting it. The TMD properties can also be periodically checked to assess its long-term durability and to foresee tuning or maintenance works. Finally, although this methodology is mainly focused on installed TMDs, it could also be extended to estimating the properties of any substructure modeled as a single-degree-offreedom system.

The paper is organized as follows: in Section 2, a rapid overview of the theoretical background is provided to present some magnitudes and nomenclature; then, Section 3 is devoted to presenting both the novel two-step methodology and the mass modification technique; Section 4 shows a validation example to which the methodology is applied; finally, Section 5 sums up the main conclusions of this work. In addition, the full dataset and code required to reproduce the results of this work are available in [17].

\section{Theoretical Background}

A tuned mass damper, and any other SDOF system attached to a structure, can be conceptually modeled as shown in Figure 1. Without loss of generality, the SDOF system is oriented in the vertical direction, but it could be placed in any other direction as long as it properly couples to the response of the structure. The movement of this conceptual model, which can be subjected to an external force $f(t)$ and/or to a base acceleration $\ddot{x}_{s}$ for being attached to a lively structure, can be described by means of the expression in Equation (1), where $m_{t}, c_{t}$ and $k_{t}$ account for the physical properties of the SDOF system (moving mass, damping constant and stiffness constant, respectively). The natural frequency, $\omega_{t}$ in $\mathrm{rad} / \mathrm{s}$, and the damping ratio $\zeta_{t}$ of the isolated SDOF system can be defined so that $\omega_{t}^{2}=k_{t} / m_{t}$ and $2 \zeta_{t} \omega_{t}=c_{t} / m_{t}$. Also, the relative coordinate $\xi(t)=x_{t}(t)-x_{s}(t)$ is defined to simplify the way the base movement is accounted for. After introducing this variable change into Equation (1), it is divided by the moving mass $m_{t}$ and the definitions of the modal properties are introduced, leading to Equation (2).

$$
\begin{gathered}
m_{t} \ddot{x}_{t}(t)+c_{t}\left(\dot{x}_{t}(t)-\dot{x}_{s}(t)\right)+k_{t}\left(x_{t}(t)-x_{s}(t)\right)=f(t) \\
\ddot{\xi}(t)+2 \zeta_{t} \omega_{t} \dot{\xi}(t)+\omega_{t}^{2} \xi(t)=\frac{1}{m_{t}} f(t)-\ddot{x}_{s}(t)
\end{gathered}
$$

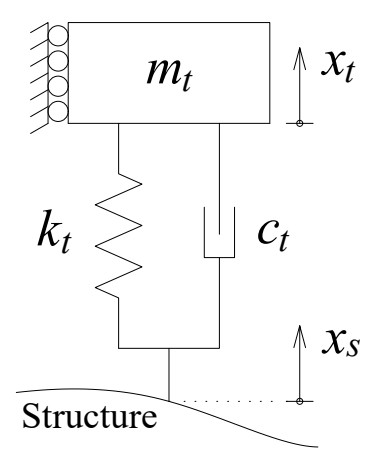

Figure 1. Conceptualization of a TMD, modeled as an SDOF system, installed on a structure.

Equation (2) is transformed into the Laplace domain by substituting the time variable $(t)$ by the complex variable $(s)$ and taking into account that $\mathcal{L}\{\ddot{\xi}(t)\}=s^{2} \Xi(s)$, $\mathcal{L}\{\dot{\zeta}(t)\}=s \Xi(s)$ and $\mathcal{L}\left\{\ddot{x}_{s}(t)\right\}=s^{2} X_{s}(s)$, where $\mathcal{L}\{\cdot\}$ is the Laplace transform operator and the uppercase functions represent the Laplace transform of the corresponding lowercase functions. Then, by letting $\omega$ be the excitation frequency in rad/s, the complex variable $s$ is substituted by $j \omega$ (so $s^{2}=-j \omega$ ), to obtain Equation (3), which is in the frequency domain. This last equation is the core of the novel methodology presented in the next section. Note that, if no force is applied $(f(t)=0 \mathrm{~N}, F(\omega)=0 \mathrm{~N})$, which is the main assumption made in [16], then the term that explicitly contains the moving mass $m_{t}$ in 
Equation (3) disappears and the transmissibility expression in Equation (4), one of the two expressions presented in [16], is derived. Also note that the structural properties are not included in this formulation, so it is not necessary for them to be known for the presented methodology to be applied.

$$
\begin{aligned}
& \left(-\omega^{2}+j 2 \zeta_{t} \omega_{t} \omega+\omega_{t}^{2}\right) \Xi(\omega)=\frac{1}{m_{t}} F(\omega)+\omega^{2} X_{s}(\omega) \\
& Y_{s \xi}(\omega)=\frac{X_{t}(\omega)-X_{s}(\omega)}{X_{s}(\omega)}=\frac{\omega^{2}}{-\omega^{2}+j 2 \zeta_{t} \omega_{t} \omega+\omega_{t}^{2}}
\end{aligned}
$$

Finally, in this work, some frequency response functions are computed from experimental data. The experimental data always consist in two time series, recorded at a certain sample rate $\left(f_{S}\right.$ in samples per second, or $\left.\mathrm{S} / \mathrm{s}\right)$. One will act as the input, $x(t)$, and the other as the output, $y(t)$. Once registered, the time domain signals may be filtered to remove any undesirable frequency components or detrended to remove any linear trend. In order to estimate the frequency response function that relates the input and the output, both time domain signals are windowed to divide the whole time series into segments of a certain size $\left(N_{t}\right)$. If required, the window function may also weight the segments to avoid frequency leakage problems. Each segment is processed separately by computing the cross power spectral density defined in Equation (5) for two signals $x(t)$ and $y(t)$, where $E\{\cdot\}$ stands for the expected value operator and the star $(*)$ accounts for the complex conjugate operand. In addition, the auto power spectral densities, $S_{x x}(\omega)$ and $S_{y y}(\omega)$, can be computed through the same expression by substituting $y(t)=x(t)$ in the first case and $x(t)=y(t)$ in the second. More details about this can be found in [18].

$$
\begin{aligned}
& S_{x y}(\omega)=\int_{-\infty}^{\infty} R_{x y}(\tau) e^{-j \omega \tau} d \tau \\
& R_{x y}(\tau)=E\left\{x(t+\tau) y^{*}(t)\right\}=E\left\{x(t) y^{*}(t-\tau)\right\}
\end{aligned}
$$

The cross and auto power spectral densities associated to every pair of processed segments can be averaged, so a final cross power spectral density, $S_{x y}(\omega)$, and a couple of auto power spectral densities, $S_{x x}(\omega)$ and $S_{y y}(\omega)$, are obtained. To calculate the frequency response function, one of the estimators shown in Equation (6) can be used, where $S_{y x}(\omega)=S_{x y}^{*}(\omega)$. The main difference between the estimators $H_{1}(\omega)$ and $H_{2}(\omega)$ is the assumption of where the noise is mostly concentrated: in the input time series or the output time series. As can be seen, the estimator $H_{v}$ is the geometric mean of $H_{1}(\omega)$ and $H_{2}(\omega)$. More details about each approach can be found in [19,20]. In this work, the $H_{v}(\omega)$ estimator is used, unless otherwise stated, and, as can be seen in the provided code [17], the function tfestimate of MATLAB is used to perform all the aforementioned estimations.

$$
\begin{aligned}
& H_{1}(\omega)=\frac{S_{x y}(\omega)}{S_{x x}(\omega)} \\
& H_{2}(\omega)=\frac{S_{y y}(\omega)}{S_{y x}(\omega)} \\
& H_{v}(\omega)=\sqrt{H_{1}(\omega) H_{2}(\omega)}=\frac{S_{x y}(\omega)}{\left|S_{x y}(\omega)\right|} \sqrt{\frac{S_{y y}(\omega)}{S_{x x}(\omega)}}
\end{aligned}
$$

\section{Methodology}

In this section, the two methodologies to estimate the moving mass are described in detail. First, the mass modification technique, a well-known methodology, is recalled here in order to provide a means to compare the obtained results; then the novel transmissibilitybased procedure is presented. 


\subsection{Mass Modification Technique}

This technique is based on the definition of the natural frequency of an SDOF system, $\omega_{t}^{2}=k_{t} / m_{t}$. The estimation of this natural frequency needs to be performed in at least two different scenarios: the first, in which the moving mass of the SDOF system is $m_{t}$; and the second one, in which an additional mass $\Delta m$ is added to it, so the new value of the moving mass is $m_{t}+\Delta m$. The stiffness value, $k_{t}$ remains unchanged, so the natural frequency varies from $\omega_{t}^{2}=k_{t} / m_{t}$ in the first scenario to $\omega_{t \Delta}^{2}=k_{t} /\left(m_{t}+\Delta m\right)$ in the second. Assuming that the added mass $\Delta m$ is known, the ratio $\omega_{t}^{2} / \omega_{t \Delta}^{2}$ permits an expression to directly estimate the value of the moving mass $m_{t}$ to be obtained, as shown in Equation (7).

$$
m_{t}=\Delta m \frac{\omega_{t \Delta}^{2}}{\omega_{t}^{2}-\omega_{t \Delta}^{2}}
$$

To apply Equation (7), both natural frequencies need to be estimated and the goodness of these estimations may compromise the final estimation of the moving mass. To mitigate the effect of small errors during the estimation of a natural frequency, more than two scenarios may be considered. In each $i$ th scenario, the moving mass is progressively increased, so $m_{t i}=m_{t}+\Delta m_{i}$, leading to a set of estimated natural frequencies, $\omega_{t i}^{2}=k_{t} / m_{t i}$. Now, the ratio $\omega_{t}^{2} / \omega_{t i}^{2}$ leads to the expression in Equation (8) which represents a straight line in the $\left(\Delta m_{i}, \omega_{t}^{2} / \omega_{t i}^{2}\right)$ plane, with a slope equal to $1 / m_{t}$.

$$
\frac{\omega_{t}^{2}}{\omega_{t i}}=1+\frac{1}{m_{t}} \Delta m_{i}
$$

In this way, several known mass increments $\Delta m_{i}$ can be added to the SDOF moving mass and the corresponding modified natural frequencies $\omega_{t i}$ can be estimated, leading to a certain squared frequency ratio $\omega_{t}^{2} / \omega_{t i}^{2}$. Then, these data can be fitted to the linear expression in Equation (8) to get the value of the slope and, from it, the value of the original moving mass, $m_{t}$. The different estimations of the natural frequency can be performed by applying the procedure explained in [16], consisting in fitting the experimental frequency domain data estimated by the $H_{v}(\omega)$ estimator (Equation (6)) to the transmissibility function (Equation (4)). This method has the advantage of not having to apply and measure an external force. However, for the method to be reliable and less sensitive to estimation errors, three or more estimations must be performed, which may make the overall moving mass estimation process rather long.

\subsection{Novel Procedure}

The procedure presented in this work is based on the expression in Equation (3). Its terms can be rearranged and Equation (9) can be obtained, where $Y_{s \tilde{\xi}}$, defined in Equation (4), accounts for the transmissibility function between the base and moving mass coordinates. Note that the expression in the right hand side of Equation (9) corresponds to the receptance function of an SDOF system, $h(\omega)$, i.e., the frequency response function relating an input force and the displacement response of that system. As can be seen, it is explicitly expressed in terms of its natural frequency $\omega_{t}$, its damping ratio $\zeta_{t}$ and its moving mass $m_{t}$.

$$
\frac{\Xi(\omega)}{F(\omega)}-Y_{s t}(\omega) \frac{X_{s}(\omega)}{F(\omega)}=\frac{1 / m_{t}}{-\omega^{2}+j 2 \zeta_{t} \omega_{t} \omega+\omega_{t}^{2}}=h(\omega)
$$

The application of this methodology requires the estimation of two response functions: $\Xi(\omega) / F(\omega)$ and $X_{s}(\omega) / F(\omega)$. Since they share the same input reference, the applied force $F(\omega)$, the response of both degrees of freedom, $x_{t}(t)$ and $x_{s}(t)$, can be simultaneously recorded, the relative coordinate $\xi(t)$ can be calculated, and both response functions can be estimated at the same time. The transmissibility function, $Y_{s \tilde{\xi}}(\omega)$, is also required for the left hand side of Equation (9) to be fully computed. Since, by definition, the transmissibility 
function requires no force to be applied on the point belonging to the structure, $f(t)=0 \mathrm{~N}$ (see Section 3), this function cannot be estimated along with the previous response functions. For this reason, this method must be applied in two stages. However, in contrast to the work presented in [16], the transmissibility function is not now required to estimate the modal properties of the SDOF system $\left(\omega_{t}\right.$ and $\left.\zeta_{t}\right)$, but rather to compute the left hand side of Equation (9), so only one identification or fitting process to the receptance SDOF response function needs to be carried out. This leads to a simultaneous estimation of the natural frequency $\omega_{t}$, the damping ratio $\zeta_{t}$ and the moving mass $m_{t}$ of the SDOF system.

More precisely, the proposed methodology could start by computing the transmissibility function between the moving mass of the installed TMD (or a similar device) and the point of the structure on which it is placed. As explained before, this is done by simultaneously measuring the acceleration of the moving mass and the acceleration of the structure to which the SDOF system is attached, while both points are moving due to an external action different from a force applied on the moving mass. A frequency response function is estimated (by using an estimator such as $H_{v}$, defined in Equation (6)) by treating the acceleration of the structure as the input and the relative acceleration as the output. Then, by applying a force on the moving mass, the acceleration response of the same two points is recorded again. Now, the frequency response functions relating both accelerations (outputs) and the applied force (input) need to be estimated. For this task to be properly done, it is advisable to apply the force in a way that does not modify the sought moving mass. For example, an impact hammer (or any device that externally exerts an impact on the moving mass) is suitable for this purpose; moreover, any sort of inertial shaker should be avoided, since it needs to be placed on the moving mass to induce a force on it. Finally, once the three response functions are computed, the left hand side of Equation (9) can be calculated and the receptance expression (right hand side of Equation (9)) can be fitted to the resulting complex series of data in the frequency domain. As a result, an estimation of the three values, $m_{t}, \omega_{t}$ and $\zeta_{t}$, are obtained at the same time. It is important to note that only a reduced set of time recordings is required for this methodology to be applied and no previous knowledge about the structure of the TMD is needed in advance. In this sense, the methodology could be applied to a TMD installed on any slender structure, no matter how complex it is.

\section{Validation Example}

To validate the proposed methodology, the SDOF system shown in Figure 2a is placed on the lively structure shown in Figure 2b. The SDOF system represents a TMD, but it is not perfectly tuned because it is not required for the purposes of this work. It is composed of a moving mass (letter A in Figure 2a), which is attached to its frame (B) via four slender plates and a spring $(C)$, conferring the ensemble a certain amount of stiffness. The damper (D) consists of a couple of permanent neodymium magnets, which are rigidly joined to the frame, placed very close to a copper plate, which is rigidly attached to the moving mass. As explained in [21,22], the movement of the copper plate inside the permanent magnetic field develops the so-called Eddy currents, creating an effect similar to an ideal viscous damper, since the overall reaction force can be assumed to be proportional to the relative velocity of both elements within a certain range of relative velocities. By means of a precise weighting scale, the total mass of the moving elements can be estimated. To do so, apart from the moving mass itself (A), half of the mass of the elastic members (C) and the mass of the magnets (D) are also included, leading to $32.52 \mathrm{~kg}$. Note that this value may not be fully representative of the effective moving mass due to the dynamic coupling effects that arise when the SDOF system is installed on the structure.

The ensemble is simply placed on the structure and the frame is designed so it can be assumed to be rigid enough. The lively structure on which the SDOF system is installed is a timber plate with a main span of $13.5 \mathrm{~m}$, a width of $1 \mathrm{~m}$ and a height of $0.14 \mathrm{~m}$. It is made of laminated wood, Glulam $24 \mathrm{~h}$. It is simply supported at both ends and, to alleviate the deflection due to its own weight, the middle section is supported by two 
elastic members made of three springs each. The first mode of this plate is at $2.33 \mathrm{~Hz}$, with a modal damping ratio of $0.27 \%$ and a mode shape similar to the first bending mode shape of a simple-supported beam. The generalized mass of that mode at the center section (the place on which the SDOF system will be installed) is $433 \mathrm{~kg}$. Note that the data given in this paragraph is intended to fully present the structure on which the TMD is placed; however, as mentioned above, it is not required for the presented methodology to be applied.

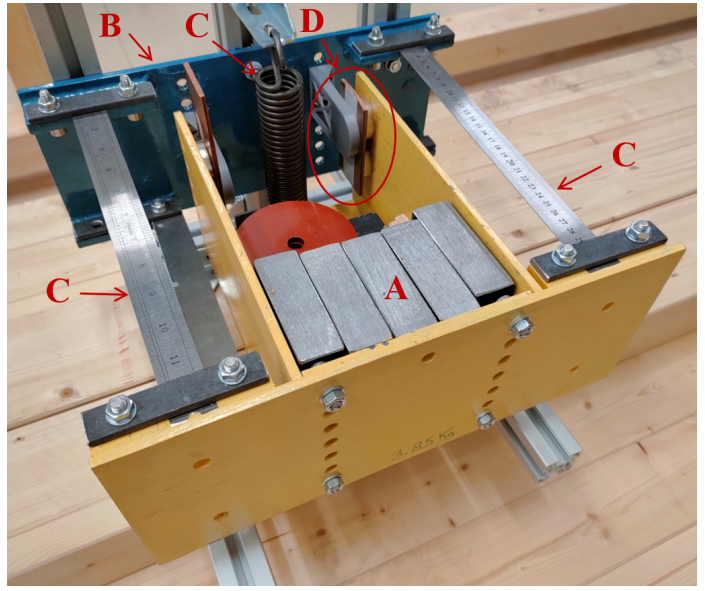

(a)

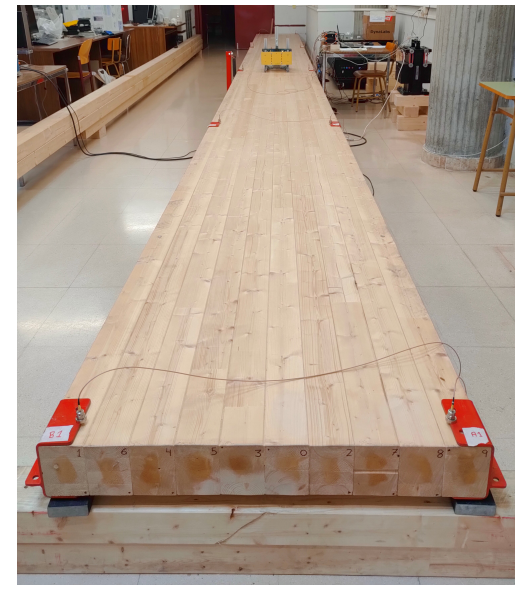

(b)

Figure 2. (a) Picture of the SDOF system used to illustrate the application of the methodologies, where $\mathrm{A}$ is the moving mass, $\mathrm{B}$ is the frame, $\mathrm{C}$ are the elastic members and $\mathrm{D}$ is the mangetic damper; and (b) the structure on which it is installed.

The example is divided into three parts. First of all, the dynamic properties of the SDOF system are identified by performing a regular experimental modal analysis in the reference scenario with it placed on a rigid floor. Then, the SDOF system is installed on the middle section of the timber plate and, by means of the mass modification technique (Section 3.1), a first estimation of its properties is carried out. Finally, the procedure presented in Section 3.2 is applied to obtain a second estimation of the same properties. Note that all recorded data, as well as the MATLAB code that processes it, is available at a Mendeley Data repository [17]. As mentioned before, the built-in function tfestimate is used to perform the estimation of the experimental frequency response functions by using the estimators $H_{1}$ and $H_{2}$ (see Equation (6)), which are then used to calculate the $H_{v}$ estimator and perform further computations. This function handles the windowing and averaging processes typical of this sort of time-to-frequency-domain transforms. On the other hand, the different curve fittings are carried out by means of the function fmincon, a built-in optimization function. A function error, which calculates the squared error between the experimental FRFs and the synthesized ones for a certain set of properties. The optimization function, then, finds the set of properties that minimizes that error.

\subsection{Isolated System}

To obtain a precise estimation of the SDOF system properties, it is first placed on a rigid floor (isolated from the structure) and, by means of an impact hammer, a force is applied to the moving mass. Both the input force and the acceleration response of the moving mass are synchronously recorded at a sample rate of $400 \mathrm{~S} / \mathrm{s}$. A total of five impacts are recorded and processed by means of the tfestimate function of MATLAB to estimate the frequency response function $\left(H_{v}\right.$ estimator). A rectangular window (i.e., with a constant weighting function equalling 1 ) with a length of $N_{t}=32,768$ points, or $81.92 \mathrm{~s}$, is used to divide the time series data into segments, so each resulting segment encompasses one single impact. The estimated accelerance (frequency response function between an input force and an acceleration response), which is computed between 0.7 and $4.5 \mathrm{~Hz}$ with a frequency resolution of $0.01 \mathrm{~Hz}$, is shown in Figure 3 (gray line). The vertical 
lines between 1.5 and $2 \mathrm{~Hz}$ correspond to very small variations around $\pi$ rad which arise due to estimation errors and noise. Since the phase plot shows data in the range $\pm \pi \mathrm{rad}$, phase values over $\pi$ rad or under $-\pi$ rad are shifted by $2 \pi$ to enter the plotting range, leading to vertical lines joining them. To estimate the SDOF system properties, the obtained complex series of data in the frequency domain is fitted to the accelerance SDOF expression shown in Equation (10), which is obtained by deriving the receptance function $h(\omega)$ in the frequency domain twice, equivalent to multiplying it by $(j \omega)^{2}=-\omega^{2}[20]$.

$$
a(\omega)=-\omega^{2} h(\omega)=\frac{-\omega^{2} / m_{t}}{-\omega^{2}+j 2 \zeta_{t} \omega_{t} \omega+\omega_{t}^{2}}
$$

Figure 3 also shows the fitted frequency response function (black line) and the corresponding estimated properties are summarized in the first row of Table 1, associated to the case "Rigid floor", which is assumed to be the reference scenario in terms of error estimation. Note that the fitted line near $\pi$ rad (under $2 \mathrm{~Hz}$ ) is actually close to the experimental data at $-\pi$ rad due to the $2 \pi$ phase shift mentioned above.

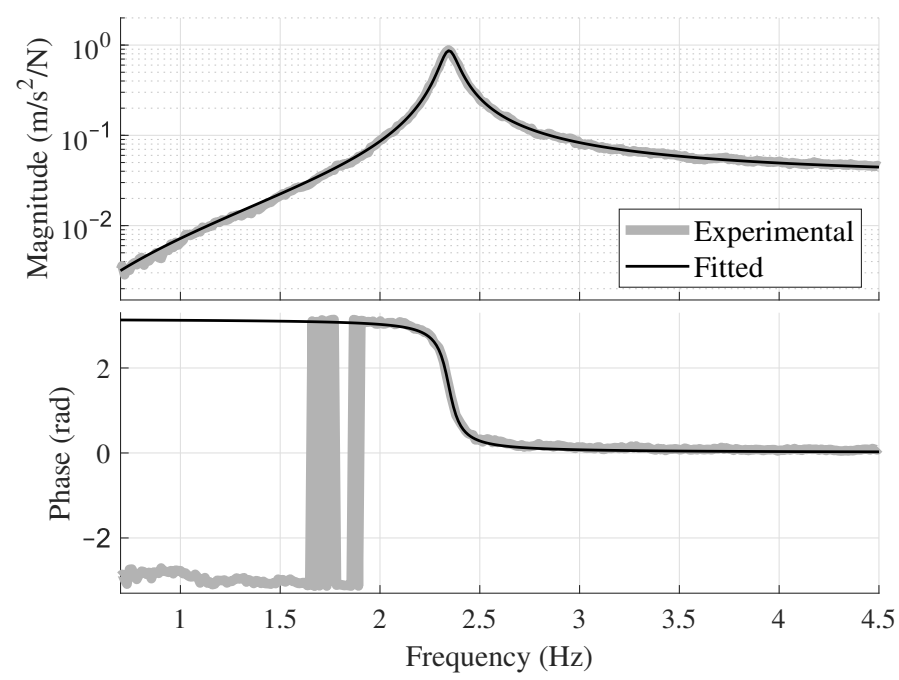

Figure 3. Fitted frequency response function of the isolated SDOF system.

Table 1. Estimated properties of the SDOF system in the three presented cases.

\begin{tabular}{ccccccc}
\hline Case & $\left.m_{t} \mathbf{( k g}\right)$ & $\boldsymbol{\epsilon}_{m}(\mathbf{\%})$ & $f_{t}(\mathbf{H z})$ & $\boldsymbol{\epsilon}_{f}(\mathbf{\%})$ & $\zeta_{t}(\%)$ & $\epsilon_{\zeta}(\%)$ \\
\hline Rigid floor & 30.84 & - & 2.345 & - & 1.87 & - \\
Mass modification & 34.52 & 11.9 & 2.340 & 0.20 & 2.01 & 7.48 \\
Novel method & 31.85 & 3.27 & 2.342 & 0.12 & 1.99 & 6.42 \\
\hline
\end{tabular}

\subsection{Mass Modification Technique}

The device is then placed on the structure. The mass modification technique is applied by considering three different scenarios: the original scenario in which the moving mass equals $m_{t}$ and no extra mass is added $\left(\Delta m_{0}=0 \mathrm{~kg}\right)$, named scenario S0; a situation in which a mass of $\Delta m_{1}=2.02 \mathrm{~kg}$ is added to it, (scenario S1); and a scenario in which a total mass of $\Delta m_{2}=4.04 \mathrm{~kg}(2 \times 2.02 \mathrm{~kg})$ is added, (scenario S2). The added mass represents $6.55 \%$ of the original moving mass in the first scenario and $13.10 \%$ in the second one. In each case, the structure is excited by walking on it (thus, not applying any direct force on the moving mass of the SDOF system, as explained in Section 3.2) and the acceleration of the moving mass $\left(\ddot{x}_{t}(t)\right)$ is synchronously recorded, together with the acceleration of the point of the structure on which the SDOF system is located $\left(\ddot{x}_{s}(t)\right)$. The relative acceleration, $\ddot{\xi}(t)=\ddot{x}_{t}(t)-\ddot{x}_{s}(t)$ is then calculated. The transmissibility function between $\ddot{x}_{s}(t)$ (input) and $\ddot{\zeta}(t)$ is estimated via the $H_{v}$ estimator in a similar way to that explained in Section 4.1. This time, however, the time series data are segmented by means of the Hanning window 
of $2^{15}=32,768$ points, instead of the rectangular one, as it is a long continuous recording. An overlapping of $2^{14}=16,384$ points (50\%) between two consecutive segments is used to overcome some problems that may arise due to the low weighting values at both window ends. The time series recording lasts around $425 \mathrm{~s}(170,000$ points at $400 \mathrm{~S} / \mathrm{s})$, so a total of 9 averages can be computed by means of the tfestimate function. The resulting transmissibility functions in the three scenarios are shown in Figure 4 (thick lines).

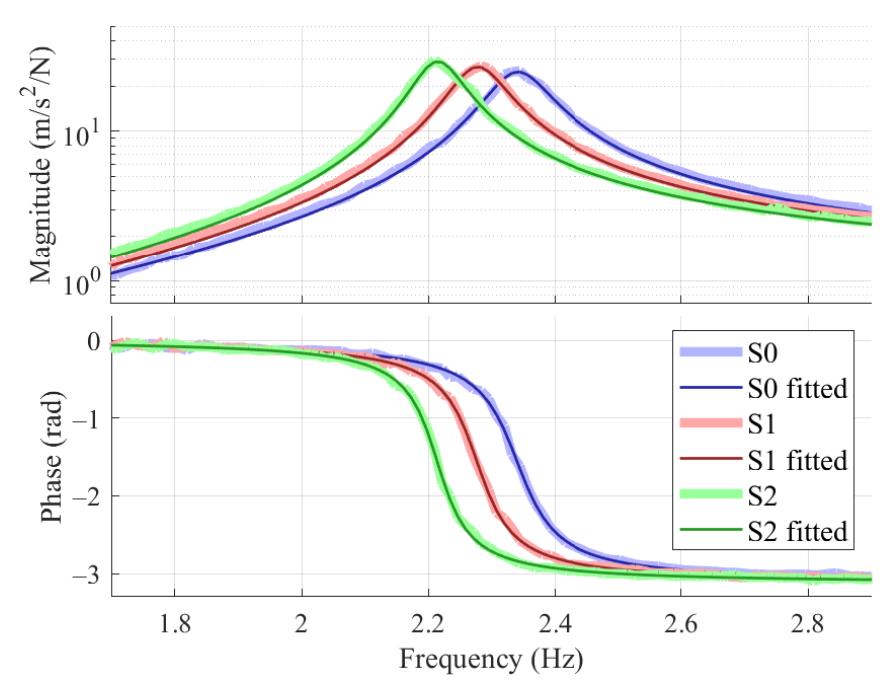

Figure 4. Experimental transmissibility functions (thick lines) and fitted curves (thin lines) associated to the three scenarios S0 (blue), S1 (red) and S2 (green).

The three transmissibility functions, one per scenario, are fitted to their corresponding expression (Equation (4)). The fitted curves are also shown in Figure 4 (thin lines). The set of identified natural frequencies and damping ratios are shown in Table 2 together with the mass added in each scenario $\left(\Delta m_{i}\right.$, where $i$ stands for the number of the scenario: 0,1 or 2$)$ and an estimation of the moving mass by applying Equation (7) (the scenario S0 is taken as the reference for both estimations). As explained Section 3.1, the points $f_{t 0}^{2} / f_{t i}^{2}$, which are equal to $\omega_{t 0}^{2} / \omega_{t i}^{2}$, plotted against the mass increment $\Delta m_{i}$, should draw a straight line. This plot is shown in Figure 5. As can be seen, the three points draw an almost perfectly straight line, with a slope of $0.0290 \mathrm{~kg}^{-1}$, leading to an original moving mass of $m_{t}=34.52 \mathrm{~kg}$, and an independent term above 0.999 , as expected. This moving mass value, together with the natural frequency and damping ratio of the scenario S0 (with no added mass), is included in Table 1 (row "Mass modification") for comparison purposes and the relative errors (where $\epsilon_{m}$ stands for the mass value error, $\epsilon_{f}$ for the natural frequency error and $\epsilon_{\zeta}$ for the damping ratio error) are estimated with respect to the reference case ("Rigid floor").

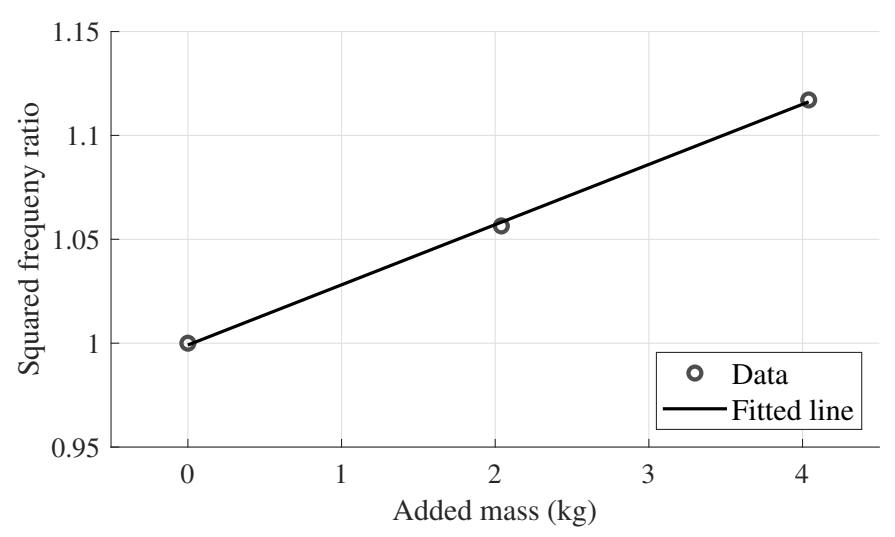

Figure 5. Squared frequency ratio $\left(f_{t 0}^{2} / f_{t i}^{2}\right.$ or $\left.\omega_{t 0}^{2} / \omega_{t i}^{2}\right)$ vs. added mass plot fitted to a straight line. 
Table 2. Estimated natural frequencies and damping ratios obtained from fitting the transmissibility function in each scenario.

\begin{tabular}{ccccc}
\hline Scenario & $\Delta m_{\boldsymbol{i}} \mathbf{( k g )}$ & $\left.f_{t i} \mathbf{( H z}\right)$ & $\zeta_{t i}(\mathbf{\%})$ & $m_{t i} \mathbf{( k g )}$ \\
\hline S0 & 0 & 2.34 & 2.01 & - \\
S1 & 2.02 & 2.28 & 1.86 & 36.15 \\
S2 & 4.04 & 2.21 & 1.71 & 34.51 \\
\hline
\end{tabular}

\subsection{Novel Procedure}

Finally, with the SDOF system installed on the lively structure, a force is applied on its moving mass, so the FRFs required to compute the left hand side of Equation (9) can be estimated. Similarly to Section 4.1, an impact hammer is used to apply the force, so the amount of moving mass is not modified during the test. The applied force is synchronously measured at $400 \mathrm{~S} / \mathrm{s}$, together with both acceleration responses (moving mass, $\ddot{x}_{t}(t)$, and structure, $\left.\ddot{x}_{s}(t)\right)$, from which the relative acceleration $\ddot{\xi}(t)$ can be computed. The time signals are divided into segments encompassing one impact each by means of a rectangular window of length 32,768 points. The resulting averaged accelerances are shown in Figure 6, where phase shifts of $2 \pi$ are also present due to noise and estimation errors.

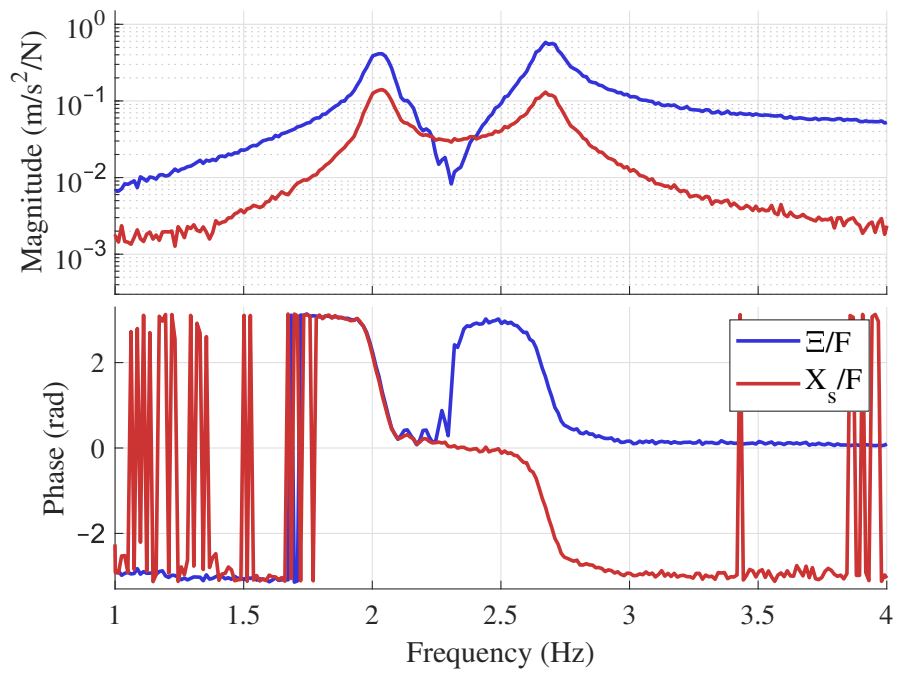

Figure 6. Accelerances associated to the relative coordinate (blue) and the structure coordinate (red) under a force applied on the moving mass.

By using these two accelerances and the transmissibility function computed in Section 4.2 as scenario S0, the left hand side of Equation (9) can be calculated. Note that all the FRFs in Equation (9) are defined as receptances, but they can be transformed into accelerances by multiplying them by $-\omega^{2}$ (second derivative in the frequency domain). As a result, the expression in the right hand side becomes the SDOF accelerance, $a(\omega)$, shown in Equation (10). Figure 7 shows the obtained curve associated to the left hand side of Equation (9), together with the accelerance estimated in Section 4.1, by isolating the SDOF system from the structure. As can be seen, there exists a high correspondence between both curves, so the left hand side of Equation (9) effectively accounts for the SDOF FRF, as expected. The dotted line in Figure 7 shows the fitting performed to the calculated curve and the resulting properties are summarized in the last row of Table 1 together with the relative error. As can be seen, a high correspondence exists between the three methods, although the novel methodology leads to a better estimate of the moving mass value in comparison with both versions of the mass modification technique. 


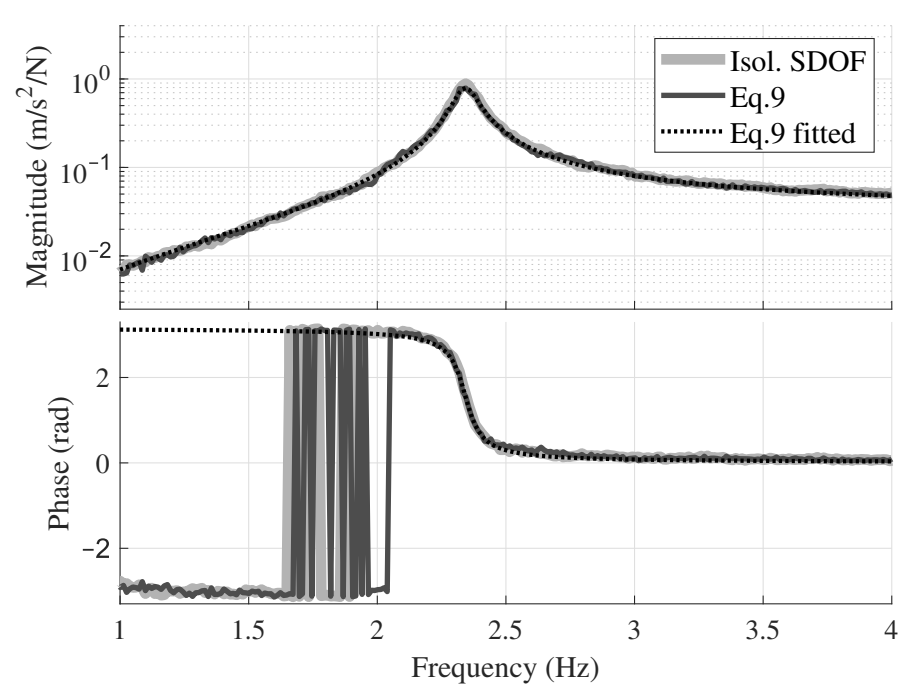

Figure 7. Comparison between the accelerance computed with the isolated SDOF system (gray line), the left hand side of Equation (9) (continuous black) and its fitting to the analytical expression (dotted).

\section{Conclusions}

As a continuation of [16], in which the authors presented a methodology based on the transmissibility function to estimate the natural frequency and damping ratio of an SDOF system installed on a lively structure, this work has been devoted to presenting a novel methodology to estimate its moving mass. It is also based on estimating the transmissibility function between the moving mass of the SDOF system and the point of the structure to which it is attached; however a second step is required in which a couple of accelerances are estimated by applying a force on the moving mass. The three FRFs take part in a rapid computation that leads to a curve that can be fitted to the analytical expression of an isolated SDOF accelerance to estimate its properties. In addition, and for comparison purposes, the mass modification technique has been presented. As shown, the presented methodology constitutes a better choice with relation to other procedures such as the mass modification technique, which, in its most robust version, requires more than two response functions to be estimated and processed, thus involving more experimental time and computational effort to provide similar or worse estimates.

As can be seen in Table 1, both methodologies provide similar estimations for the natural frequencies with very low relative errors. The damping ratio value, which is always the magnitude with the highest uncertainty, estimated by means of the mass modification technique is only slightly better, but the novel methodology provides a clearly better estimation of the moving mass with lower effort. Although, in this work, $13.10 \%$ of the original moving has been added to it, the estimation made by means of the mass modification technique could improve if more scenarios, in which more mass is added, were considered, leading to an even longer process.

Author Contributions: Conceptualization and methodology, A.M.; methodology and software, C.P. and A.I.-P.; validation, A.M., C.P. and A.I.-P.; formal analysis and investigation, A.M.; resources, C.P.; data curation, C.P. and A.I.-P.; writing—original draft preparation and editing, A.M.; writing—review, C.P., A.I.-P. and A.L.; visualization, A.M.; supervision, project administration and funding acquisition, A.L. All authors have read and agreed to the published version of the manuscript.

Funding: This research was funded by the Ministerio de Economía y Competitividad, Spanish Government, through the research project number RTI2018-098425 and the Ministerio de Educación, Cultura y Deporte, Spanish Government, through the predoctoral grant number FPU16/01339.

Institutional Review Board Statement: Not applicable.

Informed Consent Statement: Not applicable. 
Data Availability Statement: The data presented in this study are openly available in Mendeley Data at 10.17632/xx46xthc5z.1, reference number [17] (accessed date: 3 April 2021).

Conflicts of Interest: The authors declare no conflict of interest.

\author{
Abbreviations \\ FRF Frequency response function \\ SDOF Single degree of freedom \\ TMD Tuned mass damper
}

The following abbreviations are used in this manuscript:

\title{
References
}

1. Rahimi, F.; Aghayari, R.; Samali, B. Application of Tuned Mass Dampers for Structural Vibration Control: A State-of-the-art Review. Civ. Eng. J. 2020, 6, 1622-1651. [CrossRef]

2. Gutierrez Soto, M.; Adeli, H. Tuned Mass Dampers. Arch. Comput. Methods Eng. 2013, 20, 419-431. [CrossRef]

3. Jaafari, C.; Mohammadi, J. Floor Vibration Control as a Serviceability Requirement in Design Standards and Practices: Review. Pract. Period. Struct. Des. Constr. 2018, 23, 04018003. [CrossRef]

4. Ghaedi, K.; Ibrahim, Z.; Adeli, H.; Javanmardi, A. Invited Review: Recent developments in vibration control of building and bridge structures. J. Vibroeng. 2017, 19, 3564-3580. [CrossRef]

5. Soria, J.M.; Díaz, I.M.; Pereira, E.; García-Palacios, J.H.; Wang, X. Exploring vibration control strategies for a footbridge with time-varying modal parameters. J. Phys. Conf. Ser. 2016, 744, 012170. [CrossRef]

6. Xie, F.; Aly, A.M. Structural control and vibration issues in wind turbines: A review. Eng. Struct. 2020, 210, 110087. [CrossRef]

7. Belver, A.V.; Magdaleno, Á.; Brownjohn, J.M.W.; Lorenzana, A. Performance of a TMD to Mitigate Wind-Induced Interference Effects between Two Industrial Chimneys. Actuators 2021, 10, 12. [CrossRef]

8. Elias, S.; Matsagar, V. Research developments in vibration control of structures using passive tuned mass dampers. Annu. Rev. Control 2017, 44, 129-156. [CrossRef]

9. Connor, J. Introduction to Structural Motion Control; Prentice Hall Pearson Education: Upper Saddle River, NJ, USA, 2003.

10. Ormondroyd, J.; Den Hartog, J. The theory of the dynamic vibration absorber. Trans. Am. Soc. Mech. Eng. 1928, 50, 9-22.

11. Kareem, A.; Kline, S. Performance of Multiple Mass Dampers under Random Loading. J. Struct. Eng. 1995, 121, 348-361. [CrossRef]

12. Ubertini, F. Prevention of suspension bridge flutter using multiple tuned mass dampers. Wind Struct. Int. J. 2010, 13, 235-256. [CrossRef]

13. Yuan, M.; Sadhu, A.; Liu, K. Condition assessment of structure with tuned mass damper using empirical wavelet transform. $J$. Vib. Control 2018, 24, 4850-4867. [CrossRef]

14. Hazra, B.; Sadhu, A.; Lourenco, R.; Narasimhan, S. Re-tuning tuned mass dampers using ambient vibration measurements. Smart Mater. Struct. 2010, 19, 115002. [CrossRef]

15. Hwang, J.S.; Kim, H.; Kim, J. Estimation of the modal mass of a structure with a tuned-mass damper using H-infinity optimal model reduction. Eng. Struct. 2006, 28, 34-42. [CrossRef]

16. Magdaleno, A.; Lorenzana, A. A transmissibility-based procedure to estimate the modal properties of an on-board tuned mass damper. Mech. Syst. Signal Process. 2020, 135, 106378. [CrossRef]

17. Magdaleno, A.; Pelaez, C.; Iglesias-Pordomingo, A.; Lorenzana, A. Dataset for: "On the estimation of the moving mass of a TMD installed on a lively structure". Mendeley Data 2021. [CrossRef]

18. Miller, S. Probability and Random Processes, 1st ed.; Academic Press: Cambridge, MA, USA, 2012; p. 522.

19. Maia, N.; Silva, J. Theoretical and Experimental Modal Analysis; Engineering Dynamics Series; Research Studies Press: Hertfordshire, UK, 1997.

20. Ewins, D.J. Modal Testing: Theory, Practice, and Application, 2nd ed.; Research Studies Press: Hertfordshire, UK, 2000.

21. Bae, J.S.; Hwang, J.H.; Roh, J.H.; Kim, J.H.; Yi, M.S.; Lim, J.H. Vibration suppression of a cantilever beam using magnetically tuned-mass-damper. J. Sound Vib. 2012, 331, 5669-5684. [CrossRef]

22. Bourquin, F.; Caruso, G.; Peigney, M.; Siegert, D. Magnetically tuned mass dampers for optimal vibration damping of large structures. Smart Mater. Struct. 2014, 23, 085009. [CrossRef] 